\title{
ENTWARNUNG
}

\section{Ovarialzysten sind kein Krebsrisiko}

— Frauen in der Postmenopause, die Ovarialzysten haben, müssen sich nicht sorgen: Das Risiko, an einem Ovarialkarzinom zu erkranken, ist für sie nicht größer als für andere postmenopausale Frauen. Auch Mamma- und Endometriumkarzinomrisiko bleiben davon unbeeinflusst.

Zu diesem beruhigenden Ergebnis kamen Britische Wissenschaftler in einer kürzlich großen prospektiven Kohortenstudie mit 48230 Probandinnen. Die Frauen, alle zwischen 50 und 74 Jahre alt, wurden einmal jährlich sonografisch untersucht. Bei der Erstuntersuchung zwischen 2001 und 2006 fand man bei 1234 (2,5\%) Frauen eine Inklusionszyste (IC) am Ovar, 22914 hatten unauffällige Ovarien. Nach einer durchschnittlichen Gesamtbeobachtungszeit von 6,13 Jahren waren von den 1234 Frauen mit IC bei der Erstuntersuchung vier an einem Ovarialkarzinom $(\mathrm{OC})$, sieben an einem Endometriumkarzinom (EC) und 22 an Brustkrebs (BC) erkrankt. In der Vergleichsgruppe waren es 32 (OC), 90 (EC) und 397 (BC). Das relative Risiko einer späteren Diagnose für eine der drei Tumorentitäten steigt demnach mit dem Nachweis einer Ovarialzyste nicht (OC: RR 2,32; Cl, 0,86-6,28; EC: RR 1,92; Cl 0,68-3,05; BC: RR 1,12; Cl 0,73-1,73).

Für die Autoren steht nach dieser Studie fest, dass Inklusionszysten nicht der Ursprung für ein späteres Ovarialkarzinom sein können. Vielmehr nimmt das Ovarialkarzinom seinen Ursprung in anderen Beckenorganen, etwa dem Endometrium oder den Eileitern, und greift erst anschließend auf die Ovarien über.

DK .

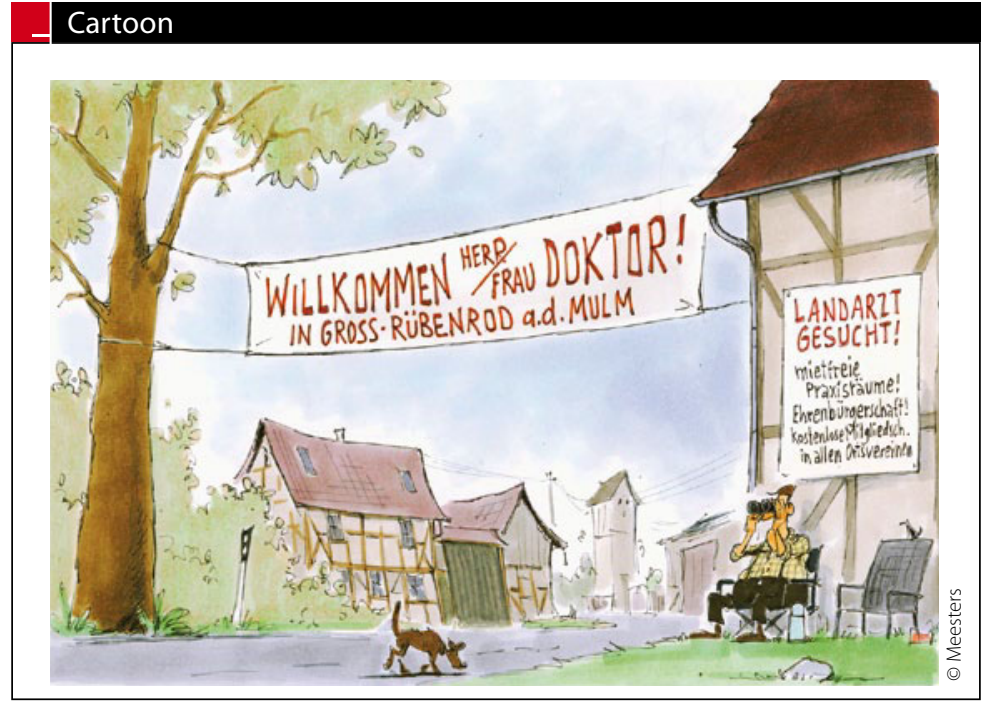

\section{AGITIERTER DEMENZPATIENT}

\section{Schmerztherapie als Beruhigungsmittel}

- Wenn demenzkranke Pflegeheimpatienten ruhelos umherwandern oder randalieren, werden sie meistens mit Antipsychotika ruhiggestellt. Eine Optimierung der Schmerztherapie könnte oft die bessere Lösung sein. Mit einem von der American Geriatric Society empfohlenen Stufenplan für die Schmerztherapie gelang es in norwegischen Pflegeheimen, auch Agitiertheit und Aggression von Demenzkranken deutlich zu bessern.

352 Heimpatienten mit mittelschwerer bis schwerer Demenz und Verhaltensstö- rungen hatten entweder die übliche Versorgung erhalten oder zusätzlich jeden Tag eine individuelle Schmerztherapie. Nach acht Wochen gaben die Patienten der Interventionsgruppe erwartungsgemäß signifikant weniger Schmerzen an als die Patienten der Kontrollgruppe. Sie waren außerdem auch weniger agitiert. Nach dem Absetzen der Schmerztherapie nach Woche acht verschlechterte sich dieser Zustand wieder.

BS Husebo BS et al. BMJ 2011;343:d4065
WER GUTES ERWARTET ...

\section{... wird seltener vom Schlag getroffen}

— Je positiver ein Mensch in die Zukunft blickt, umso eher bleibt er vom Schlaganfall verschont. Dies schließen Psychologen und Ärzte der University of Michigan aus prospektiv erhobenen Daten der Health and Retirement Study. Beteiligt waren 6044 Personen, die zu Studienbeginn noch keinen Schlaganfall erlitten hatten.

Im Beobachtungszeitraum von zwei Jahren wurden 88 Schlaganfälle erfasst. Eine optimistische Einstellung erwies sich als protektiver Faktor, der sogar eine dosisabhängige Wirkung zeigte: Mit jedem Punkt mehr auf der von 3 bis 18 reichenden Optimismus-Skala sank das Insultrisiko um jeweils $9 \%(p<0,01)$. Studienerstautor Eric Kim vermutet, dass Menschen, die sich Gutes vom Leben erwarten, auch mehr für ihre Gesundheit tun. Es gebe aber auch Hinweise, dass positives Denken direkte biologische Auswirkungen habe. „Ähnlich wie eine Depression körperliche Funktionen ungünstig beeinflussen kann, kannsich offensichtlich umgekehrt Optimismus günstig auf die Gesundheit auswirken", so Kim.

$\mathrm{BS}$.

Kim ES et al. Stroke 2011, online July 22 